\title{
Immersive Virtual Tour using Multi-projection with Remote Cluster Rendering
}

\author{
Zhijie Lin $^{1,2 \dagger}$, LeiZhao ${ }^{1}$, Qingshu Yuan ${ }^{3}$, Dongming Lu ${ }^{1}$,Yuqi Li ${ }^{1}$ \\ 1: Zhejiang University, Hangzhou, China \\ 2: Zhejiang University of Science and Technology, Hangzhou, China \\ 3: Hangzhou Normal University, Hangzhou, China
}

\begin{abstract}
This paper introduces a new virtual tour method which integrates multiprojection techniques, remote cluster rendering and pattern tracking. We use geometric calibration and edge blending to create a seamless and consistent display and utilize remote render cluster to render high-resolution scene in real time through China Next Generation Internet (CNGI). By tracking the hand-held pattern, users can control the 6 degrees of freedom (6DOF) of virtual tour. We implement a virtual tour application referring to "Dunhuang Grottos". The successful demonstration means that we can provide a real-time convenient rendering service for virtual tour in remote museums.
\end{abstract}

Keywords- multi-projection, remote render cluster, virtual tour

\section{INTRODUCTION}

Today, a variety of immersive multi-projection displays have been applied to present digital resources of cultural relics (e.g. Dunhuang Grottos). Tiling multiple projectors to create a single display with high-resolution and wide field-of-view, the immersive virtual reality (VR) environment increases the sense of immersion experienced by users. Take full advantages of the immersive display, multiprojection techniques provides interactive virtual tours for users. In the mutliprojection system, PC-Cluster is used for realistic and high resolution image generation. [1] proposes a self-distributing software environment for PC-Clusterbased immersive projection displays to achieve a low-latency with $100 \mathrm{Mbps}$ Ethernet, and [2] proposes a tele-immersion environment system which uses ultrahigh-speed network(Japan Gigabit Network 2) to interconnect highly immersive systems such as CAVE distributed at various places in Japan. Unlike [1], our method is composed of two parts, projection subsystem and remote cluster rendering subsystem, they are not coupled together, can be placed in different place and connected by high speed network. [2] is much similar to our method, the rendered image is transferred to projection end by Japan Gigabit Network 2, which is a kind of high speed network. But the most difference is that we use ray tracing method to render the scene to generate highly realistic result image.

The multi-projection with remote rendering system is useful in some case. For example, in the museum exhibition, high-resolution image and 3D model of cultural relics is so precious that they can't be easily copied to other institution because of copyright restriction and lack of trust. Another reason is that some 3D

†E-mail: bytelin@qq.com, corresponding author 
models are so large that the render tasks to produce a high quality image can't be achieved within real-time especially in interactive case if without cluster rendering. At the same time, the good condition for remote cluster rendering has been created on the strength of high speed network IPv6.

With the power of highly realistic rendering cluster, our system can provide rendering service to some institutions like museum, EXPO and so on. The only thing they should do is to install our projection subsystem and connect them to the network.

There are three primary issues involved in building our virtual tour multiprojection system.

(a) Calibration for multi-projection display. Based on geometric calibration, the overall display distortion caused by irregular-shape surfaces and arbitrary alignment of projectors can be corrected [3]. Moreover, color calibration solves the inconsistency problems of chrominance and luminance in projection. [4]

(b) Real-time rendering. Real-time high-quality rendering is necessary for a virtual tour system; however, the displayed scenes in immersive environment are typically very large. Render cluster with highperformance can deal with these problems. [5]

(c) Interactive method. It seems positively unnatural to control the 6DOF with traditional GUI devices (e.g. Joystick). [6] Hence other novel interactive methods are applied to virtual tour system.

The paper begins by introducing our system architecture and giving an overview of our application. Next, we focus on the last two issues mentioned above and describe the details of our solution. The first issue has been solved in our previous work. $[3,7,8,9]$ we will not talk about them in details in this paper. Then, we present our application of virtual tour in Dunhuang grottos. Finally, we discuss the performance of our application and propose some future enhancements.

\section{TECHNICAL DETAILS OF OUR FRAMEWORK}

\section{A. Framework Architecture}

As shown in Fig. 1, to setup a universal framework of our virtual tour system, we adopt the $\mathrm{C} / \mathrm{S}$ structure. The remote render cluster provides high-resolution rendering service to client multi-projection system which may be implemented in exhibition halls and museums. Benefit from the high bind-width of CNGI, rendering results can be transmit from render sever to projection sever with less latency or packet loss. The multi-projection server receives $2 \mathrm{D}$ frames and forwards them to projection nodes. To create a single seamless display, each node rewraps and blends frames according to the alignment of projectors. Working as an interactive device, a web camera capture the pattern held by users. Then our system can analyze and update the information of 6DOF. 


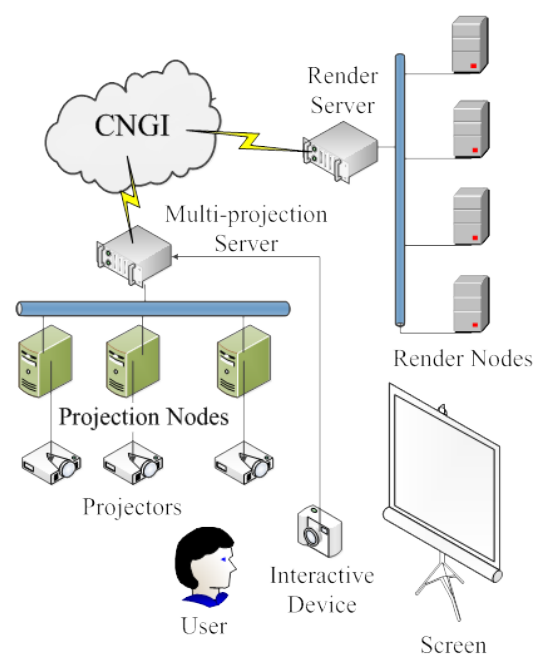

Figure 1. System Architecture

\section{B. Multi-Projection display}

We implement our virtual environment on a cylindrical screen with camerabased geometric calibration technique. Non-planar screen and non-vertical orientation of projection cause distortion in display. We use nonparametric method for piecewise linear mapping from the display coordinates to the camera coordinates. But since the images captured by camera are 2D plane, the direct view-dependent method can't be used to mapping the image to curve surface.Using one camera on a high-precision turn-plate, we can capture the screen with different direction and the same position. Through the analysis to the rotation angle and the properties of camera, the extrinsic parameters can be estimated easily. Once the extrinsic parameters have been solved, the frustums for mapping can be derived. More technical details can be found in our work[7].

Taking into account the exhibition agencies may lack professional personnel, we are also prepared an online continuous self-calibration program, when the display link configuration was changed in the careless, the system can be adaptively re-calibration and resume normal projection display. It's technical framework shown in Figure 2. Each projector bind an industrial camera as the image sensor, the camera observed the whole process of projection screen, without pre-correction. The method uses a binocular vision online extracted SURF features from the projected scene and screen edge curves, computing projector's and camera's pose parameters adaptively in real-time. 


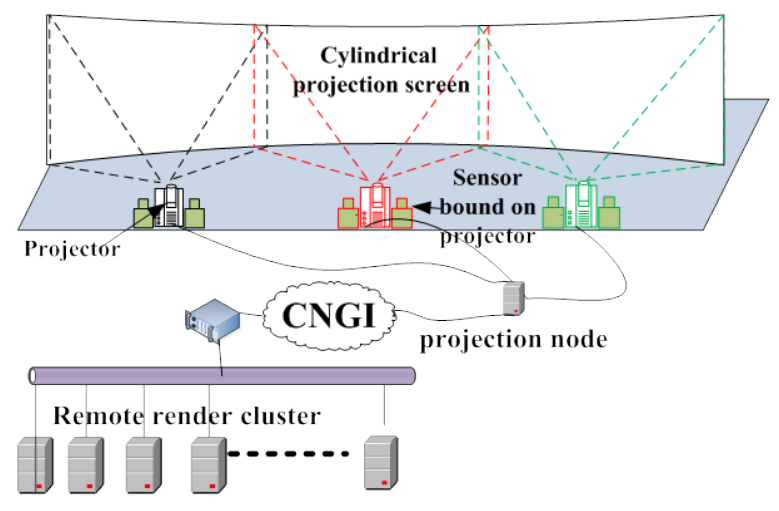

Figure 2. Online Continuous Self-calibration Projection Framework

In order to improve accuracy, we design a nonlinear constrained model, Binding the constraints of quadratic curve, reprojection, and structural vertical, parallel constraint implicit in screen surface, get the minimum error estimates. Also includes a projection area recognition algorithm, projection screen surface fitting algorithm, Kalman filter continuous correction algorithm. Based on all these methods, achieve continuous adaptive correction.

\section{Remote Cluster Rendering}

The model of our virtual scene is very large, typically with millions patches. So we utilize parallel rendering cluster to deal with the scalable task. View frustum and view position in rendering are according to the extrinsic parameters and physical shape of screen.

The most important issue in parallel rendering is load balancing in our rendering cluster. The framework of our rendering system is based on Equalizer[10], which is the standard open source library to create and deploy parallel OpenGL-based applications. We adopt the sort-first rendering architecture to for task partitioning in high-quality rendering.

The method partitions the render tasks in image space, which is suitable for ray tracing method. Every render node is responsible for one image block. And the final image is composed by these blocks after rendering process as show in Fig. 3. 


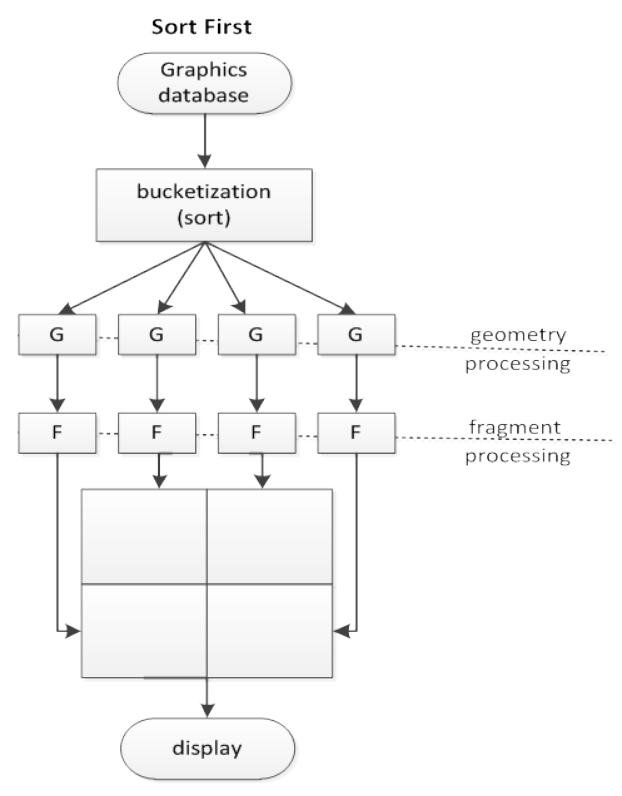

Figure 3. Sort-first ${ }^{[11]}$

The strategy for mapping screen regions to different nodes has a critical impact on the performance of a sort-first system[11]. A simple equal-dividing can result in severe load-balancing problems since the total task distribution isn't spatial uniform. Therefore, we adopt an adaptive load-balancing technique through prediction binary tree (PBT) [12]. PBT records a partition of image space. Every leaf node represents a corresponding image block. The root node can be seemed as all render tasks and all non-leaf nodes can be divided into two sub node until the number of leaf node equals to cluster render nodes. Fig. 4 shows a PBT with eight leaf nodes. We assume that temporal coherence property exists in adjacent frames. In the first time of rendering, we assume every pixel in the image space consume one unit time, and then we divide the image space into some blocks equal to the number of cluster node to initialize PBT and make sure that every leaf node has similar sum of consume time. After the render of first frame is over, the rendering system will record current task partitioning and time consume of every block. We can calculate the time consume of per pixel in every block and displace the old time. Then we use the same method to re-partition the image space and update the PBT at the beginning of every next frame rendering. Adopting the adaptive load-balancing technique, the average performance of our system has been significantly enhanced (50\% or more). 


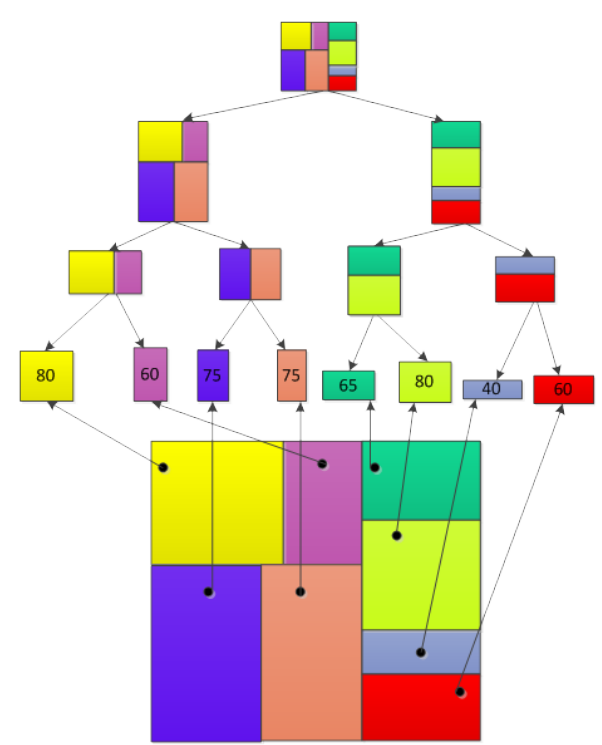

Figure 4. Prediction binary tree $\mathrm{e}^{[13]}$

Our system is mainly oriented to museum exhibition. To exhibit relics as really as possible, traditional rendering method like rasterization will not fulfill our requirement. In our system we choose ray tracing method because this method can simulate a variety of lighting effects and shadow. And this just meets our need. Additionally ray tracing method itself has very high computation amount which leads its low performance. Because our system is interactive style, we need realtime ray tracing method.

In our system, we use Nvidia's ray tracing engine OptiX based on GPU whose performance is much better than ordinary method based on CPU. At the same time, we transform the OptiX to cluster-based environment. In rendering process, we first use sort-first architecture to divide image space and then assign the tasks to every render node. Within each render node, we use GPU based ray tracing algorithm on every pixel. Because of GPU thread, the process of ray tracing on every pixel is parallel as show in Fig. 5 and Fig. 6. With the help of PBT, every render node is parallel rendered and the time consume is similar. The parallelism between nodes and within node promotes rendering performance to a great extent. 


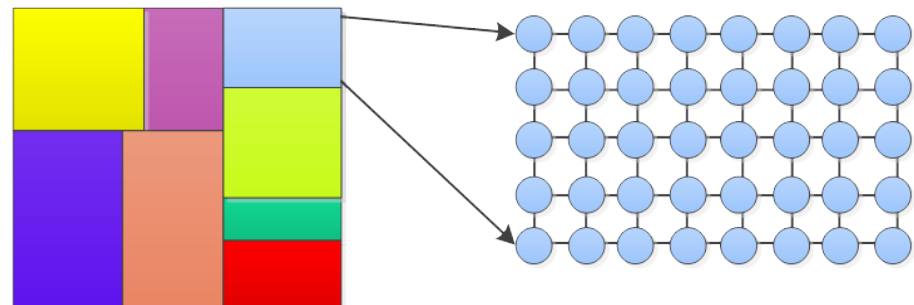

Parallel between nodes

Parallel within node

Figure 5. parallel rendering ${ }^{[13]}$

Render node

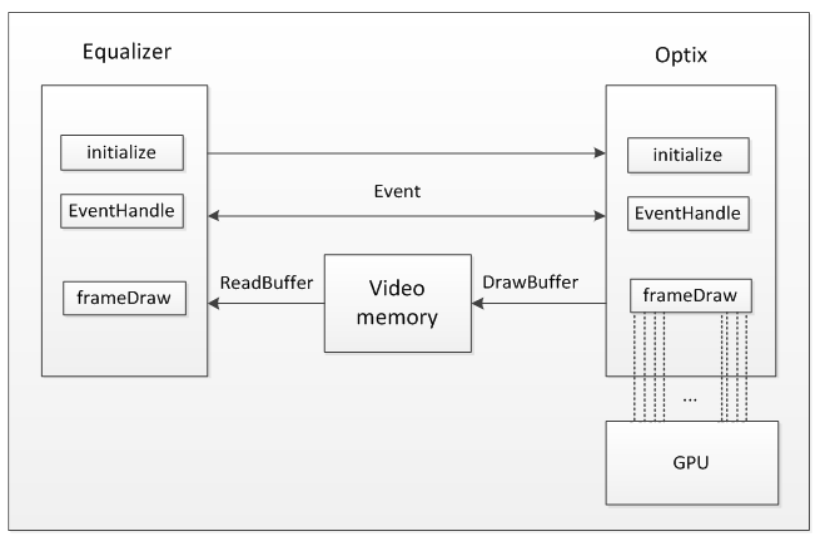

Figure 6. Communication within node

All the blocks of image will be composed and generate an entire image. The resolution of image is mainly restricted by the band-width of network and the compression ratio of rendered frames. Our system is deployed with IPv6 protocol on the CNGI and compress frames into JPEG format since the JPEG images can reach extreme high compression ratio. Fig. 7 shows a rendered frame with less quality loss received by projection server. 


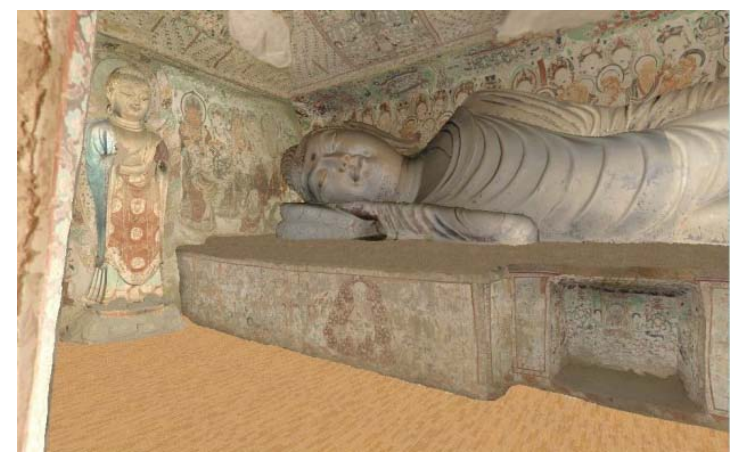

Figure 7. A rendered frame of "Dunhuang 158th grotto" received by projection server

\section{Interactive Method}

Motivated from the handheld interaction method of Augmented Reality (AR) technique, we use a handheld pattern to control the view position and view angle in virtual tour as shown in Fig. 8. Developing with ARtoolkit, the 6DOF information can be easily acquired.

Since the height of a person would not be changed in virtual tour, we set a fix value to the position in vertical direction. But it worth notice that the horizontal rotation angle of the pattern can't cover the whole degree and the horizontal position should be rescaled since the irregular bounding box. So we use the value of horizontal rotation angle to control the speed horizontal angular velocity, and use the offset value of horizontal position to control the horizontal translational speed. And we filter the high frequency to remove vibrating in pattern register.

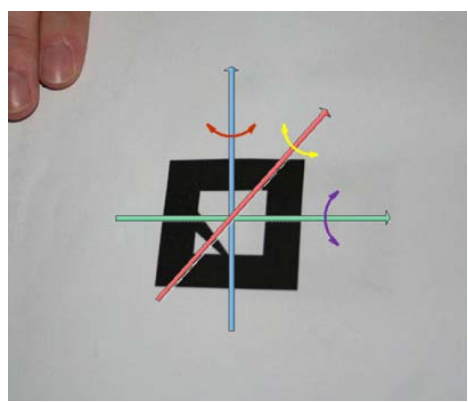

Figure 8. 6DOF analyses with captured pattern

\section{IMPLEMENTATION \& RESULTS}

Our render system consist of an admin node and 16 render nodes, each node with 4 Intel xeon e5620 2.4GHz, 12GB RAM and NVIDIA GeForce GTX 480 for rendering. The projection system consist of an admin node, 3 projection node (AMD Dual Processor $2.01 \mathrm{GHz}$, 3GB RAM, NVIDIA GeForce 6800) and three LCD projectors. The networking devices consist of two gigabit Ethernet switches connecting to CNGI and two fast Ethernet switches connecting to projection cluster 
and rendering cluster respectively. We use a web camera with resolution $\left(800^{\times}\right.$ 600) for interaction.

As shown in Fig. 10, a virtual tour application named "Dunhuang 158th grotto" which has more than 6 million triangles is implemented with our system. Users can adjust the view angle and view position by move the pattern held in their hands. The resolution of our overall display is $1920 \times 1080$. On average, the frame rate of our system can reach 20 frames/s, which can afford real-time interaction. In the application, the gigabit network utilization ratio reaches $50 \%$ or more; which prove that the high bind-width of CNGI is necessary in such high quality display.

We also evaluate the performance in regard to changes with the amount of render nodes and 3D data size. As can be seen from Fig. 9, When the 3D data size is relatively small, the performance decreases as the rendering of the number of nodes increases. However, when the 3D data size is relatively large, performance is improved with the increasing number of rendering nodes.

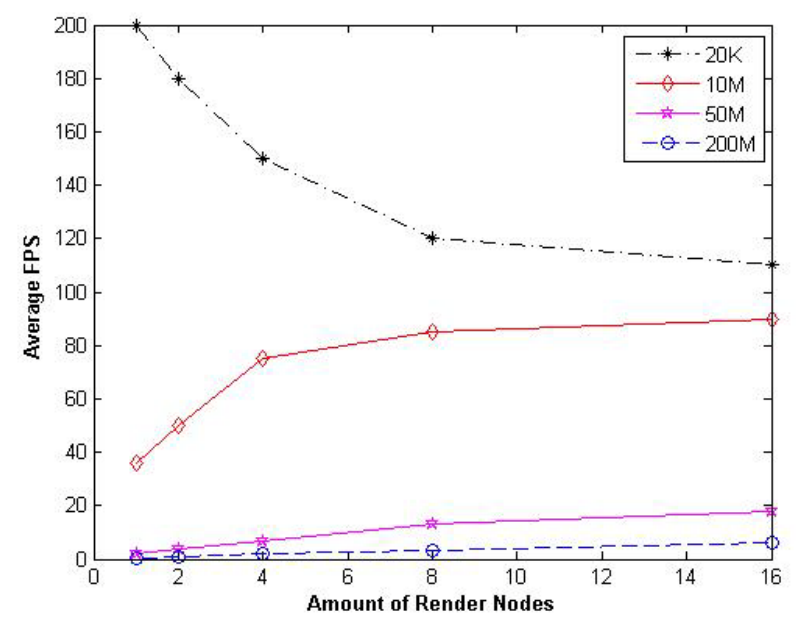

Figure 9. Rendering Performance changes with nodes number and data size 


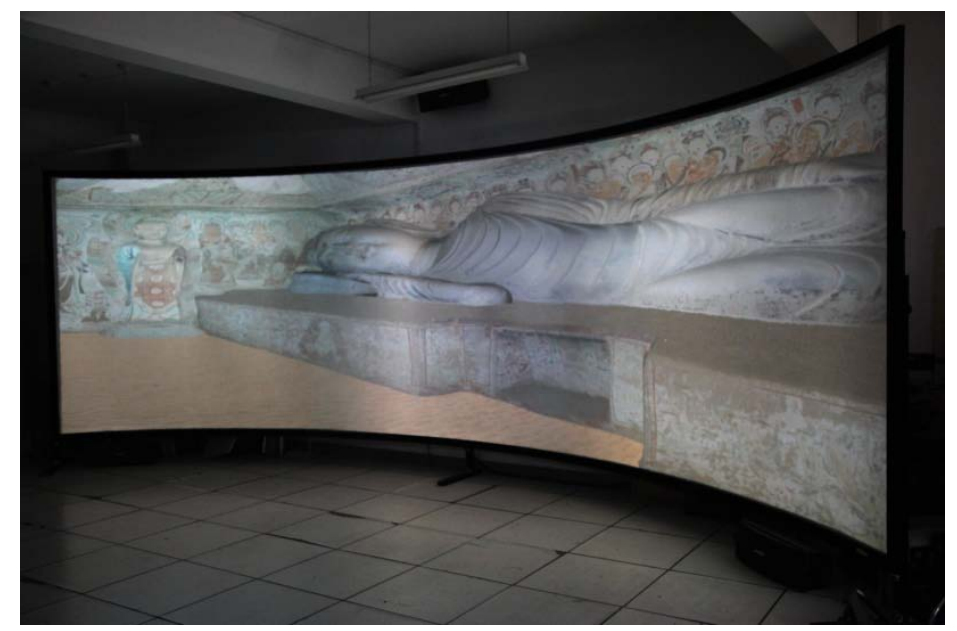

Figure 10. Our virtual tour application named "Dunhuang 158th grotto"

\section{CONCLUSION \& FUTURE WORKS}

In this paper, we have presented a virtual tour system which integrates techniques of multi-projection and remote cluster rendering. To create a seamless uniform display, we use structured light method and "two-pass render" for geometric calibration and use channels response function for color calibration. We utilize PBT to deal with the load balancing issue in scalable parallel rendering for large scene.

There are much can be enhanced in our system. It is not difficult to render the scene for two eyes, so we would like to apply stereoscopic view to our system. We will seek to extend our method to other screens with different shape. Moreover, we look forward to get rid of patterns to make natural interaction in virtual tour. We hope we could provide a convenient remote render service for museums with immersive screen in the future.

Acknowledgment : This work is supported in part by grants from National Basic Research Program of China (2012CB725305), National Natural Science Foundation of China (61003146), Provincial Natural Science Foundation of Zhejiang (LY13F020050), and Key Sci-tech Innovation Team of Zhejiang Province (2010R50040).

\section{REFERENCES}

[1] Naoki Hashimoto, Yoshihiko Ishida, and Makoto Sato.”A Self-Distributing Software Environment for Immersive Multiprojector Displays”. IEICE Transactions on Information and Systems, D (Japanese Edition), Vol.J89-D, No.2,pp.362-370, 2006

[2] Tomoyuki Ishida, Akihiro Miyakawa, Yoshitaka Shibata.”A tele-immersion environment for traditional Japanese crafting system over the Japan Gigabit Network". International Journal of Web and Grid Services,Vol.4, No.1, pp.100-116, 2008

[3] Yuan Qingshu, Lu Dongming."Multi-projector Calibration and Alignment Using Flatness Analysis for Irregular Shape Surfaces". Proceedings of Pacific Rim Conference on Multimedia, 2008,pp.436-445. 
[4] Zhang Liangjie, Jiang Xiaohong; Lei Kaibin, Xiong Hua. "Building Virtual Entertainment Environment with Tiled Display Wall and Motion Tracking”. International Conference on Virtual Reality and Visualization 2011, pp.283-286,2011.

[5] Pan Weijing, Yuan Qingshu, Lu Dongming. "Universal Browse and Display Framework for Ultra-resolution Image”, 2010 3rd IEEE International Conference on Ubi-Media Computing, U-Media 2010,PP.305-309,August 2010.

[6] Marc Pollefeys, Luc Van Gool, Ive Akkermans ,Dirk De Becker, Kris Demuynck. "A Guided Tour to Virtual Sagalassos”. Proceedings of the 2001 conference on Virtual reality, archeology, and cultural heritage pp.213-218

[7] Yuan Qingshu, Lu Dongming, He Yueming, Shi Xifan. "Multi-Projector Mosaic for Curved Screen Display System”. The 4th International Conference on Digital Heritage. Oct. 2006. Kaifeng, China

[8] Juang Ray; Majumder Aditi. Photometric Self-Calibration of a Projector-Camera System. In: IEEE Conference on Computer Vision and Pattern Recognition, pp:35443551, 2007

[9] Yuan Qingshu, Lu DM, Chen WD, Pan YH. MultiPro: A platform for PC cluster based active stereo display system. LECTURE NOTES IN COMPUTER SCIENCE, 2005, 3480,pp.865-874

[10] Eilemann Stefan, Makhinya Maxim, Pajarola Renato. Equalizer: A scalable parallel rendering framework. IEEE Transactions on Visualization and Computer Graphics, v 15, n 3, pp.436-452, May-June 2009

[11] Mueller Carl. Sort-First Rendering Architecture for High-Performance Graphics. Proceedings of the Symposium on Interactive 3D Graphics, pp. 75-84, 1995

[12] Cosenza Biagio, Cordasco Gennaro, De Chiara Rosario, Scarano Vittorio, Erra Ugo. Load Balancing in Mesh-like Computations using Prediction Binary Trees. Proceedings of the 7th International Symposium on Parallel and Distributed Computing, ISPDC 2008,pp. 139146, 2008

[13] Lei zhao,"Research on the key technologies of High Realistic Rendering Platform based on GPU Cluster", unpublished 